\title{
Differentiation of NTERA-2 Clonal Human Embryonal Carcinoma Cells into Neurons Involves the Induction of All Three Neurofilament Proteins
}

\author{
Virginia M.-Y. Lee* and Peter W. Andrews $\dagger$ \\ *Division of Neuropathology, Department of Pathology and Laboratory Medicine, The University of Pennsylvania \\ School of Medicine, Philadelphia, Pennsylvania 19104, and †The Wistar Institute of Anatomy and Biology, \\ Philadelphia, Pennsylvania 19104
}

\begin{abstract}
Monoclonal antibodies were used in indirect immunofluorescence and immunoblot studies to examine the expression of four different classes of intermediate filaments, namely, neurofilaments, glial filaments, cytokeratin, and vimentin, in NTERA-2 cl.D1 (NT2/D1) pluripotent human embryonal carcinoma (EC) cells, and in the neurons derived from these cells by differentiation induced with retinoic acid. In the EC cell cultures, grown in the absence of retinoic acid, cytokeratin was the predominant intermediate filament detected by immunofluorescence; only a few cells expressed vimentin, and none expressed glial filament protein or any of the three neurofilament proteins (NF195, NF170, and NF70). Immunoblot analyses of cytoskeletal extracts of these cells supported these data. Two days after exposure to retinoic acid, all three neurofilament subunits were detected in a few cells with a non-neuronal morphology and, by double indirect immunofluorescence, were observed to colocalize with cytokeratin. The number of neurofilament-positive cells increased with time after initial exposure to retinoic acid, and although $95 \%$ of these cells contained cytokeratin initially, less than $5 \%$ of the neurofilament-positive cells retained cytokeratin 2 weeks later. By this time, many of the cells expressing all three neurofilaments but no cytokeratin exhibited a neuronal morphology. Vimentin was evident in a large number of cells in the cultures, but it was not detected in the neurofilament-positive cells. Also, many of the neurofilament-negative cells continued to express cytokeratin. No cells expressing glial filament proteins were found. Immunoblot analysis of the differentiated cultures also revealed all three neurofilament subunits, and vimentin and cytokeratin, but no glial filament protein. We conclude that NT2/D1 EC cells, induced to differentiate with retinoic acid, provide a model system for studies of human neuronal differentiation and for the development regulation of intermediate filament expression.
\end{abstract}

Teratocarcinomas contain a variety of differentiated cell types that arise from a common stem cell, the embryonal carcinoma (EC) cell (Solter and Damjanov, 1979; Stevens, 1967). The EC cells exhibit biochemical and developmental properties similar to the cells of the early embryo, and in vitro, murine EC cells have provided a model for studying cellular differentiation in the early stages of mouse embryogenesis (Martin, 1980). Recently, we described a clonally derived, pluripotent human EC

\footnotetext{
Received Dec. 28, 1984; revised Aug. 22, 1985; accepted Aug. 23, 1985.

This work was supported by Grants CA 29894, CA 36245, and NS 18616 from the National Institutes of Health. We thank Mr. M. Dignazio for excellent technical assistance and Ms. M. Obrocka for the photographic work.

Correspondence should be addressed to Dr. Peter Andrews, at the Wistar Institute of Anatomy and Biology, 36th Street at Spruce, Philadelphia, PA 19104 Copyright (C) 1986 Society for Neuroscience $0270-6474 / 86 / 020514-08 \$ 02.00 / 0$
}

cell line, NTERA-2 cl.DI (NT2/Dl) (Andrews et al., 1984), which differentiates in response to retinoic acid and provides a corresponding model for human development (Andrews, 1984). Amongst the differentiated derivatives of this human EC cell line were cells with phenotypic properties of neurons; i.e., they expressed tetanus toxin receptors and the largest neurofilament (NF) protein, NF195.

NF proteins represent one class of intermediate filaments (IF). These are cytoskeletal structures present in most mammalian cells and include five different, immunochemically distinct classes that are distinguished by their polypeptide subunits (Lazarides, 1982). In mature neurons, NF are heteropolymers composed of a triplet of proteins (NF70, NF170, and NF1 95). However, these subunits may not be expressed to the same extent by all mature neurons (Bennett et al., 1984; Dahl, 1983; Hirokawa et al., 1984; Toyoshima et al., 1984) and tumor-derived neurons (Bergh et al., 1984; Lee and Page, 1984). Because the different IF classes are cell-type-specific and developmentally regulated, antibodies to their various subunits have been used as probes to study differentiating $\mathrm{EC}$ cells in vivo and in vitro (Damjanov and Andrews, 1983; Damjanov et al., 1984; Jackson et al., 1980; JonesVilleneuve et al., 1982; Lazarides, 1982; Paulin et al., 1982; Ramaekers et al., 1984).

We have produced a panel of monoclonal antibodies (MAs) that distinguish between the following three IF classes present in brain: NF, including each of the NF triplet proteins; glial filaments (GF), which are present in glial cells; and vimentin filaments (VF), which are present in some neurons, glial cells, and other cell types (Lee et al., 1982b, 1984). These antibodies are effective probes for in vivo and in vitro studies of developing cells (Lce and Pagc, 1984; Lee et al., 1982a; Trojanowski et al., 1984a). We have now used them to characterize further the differentiation of NT2/D1 human EC cells into neurons following retinoic acid induction in vitro. In addition, we used MAs specific for cytokeratin filament (KF) proteins, the IF of epithelial cells, since KF is the earliest class of IF expressed in embryogenesis (Jackson et al., 1980) and is expressed by human EC cells (Damjanov and Andrews, 1983; Damjanov et al., 1984). We now find that, whereas the NT2/D1 pluripotent human EC stem cells express KF, a subpopulation of their differentiated derivatives expresses all three NF subunits but none of the other classes of IF proteins. Other differentiated cells in the same cultures express VF or $\mathrm{KF}$, or both VF and $\mathrm{KF}$, while none express GF proteins.

\section{Materials and Methods}

\section{Human EC cells and their differentiation in culture}

NTERA-2 cl.D1 (NT2/D1) clonally derived human EC cells were maintained as previously described (Andrews et al., 1984). They were pas- 

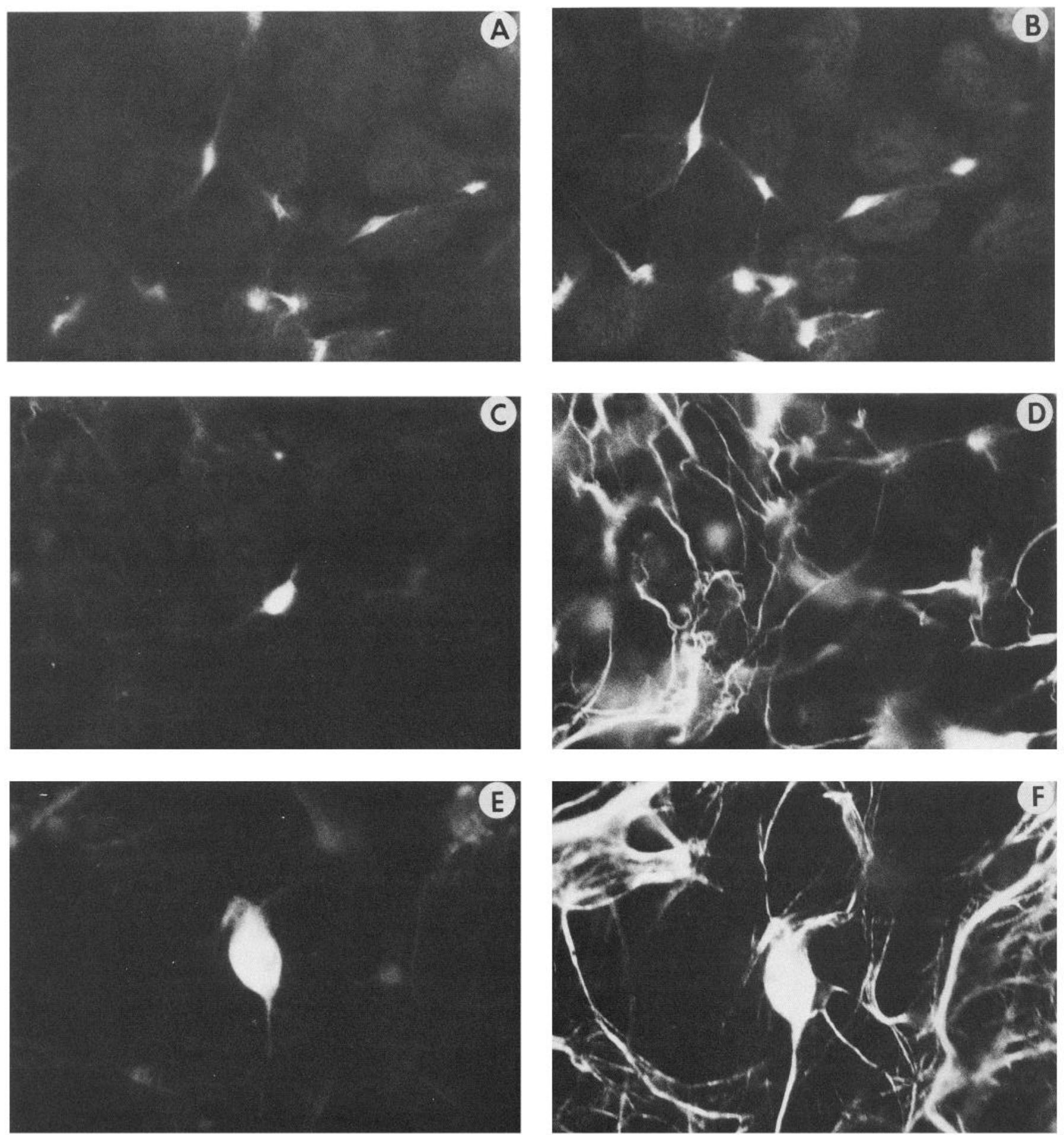

Figure 1. Double indirect immunofluorescence staining of differentiated NT2/D1 cells with anti-NF and anti-KF MAs. These cultures were obtained by exposing NT2/D1 EC cells, grown on glass coverslips, to $10^{-5} \mathrm{M}$ retinoic acid for $5 \mathrm{~d}(A, B, E$, and $F)$ or for $14 \mathrm{~d}(C, D)$. $A$, Staining of cells with a non-neuronal morphology with RMDO20, an anti-NF195 MA. B, Staining of NF170 in the same cells with HO14. C, Staining of NF170 in a cell with neuronal morphology using HO14. D. Staining of KF in the same preparation as in $C$ with Chrome 1 . Note that the NF170positive cell in $C$ is KF-negative. $E$, Staining of a cell for NF170 with HO14. F, Staining of KF in the same preparation as $E$ with Chrome 1 . Note that the NF170-positive cell in $E$ also expesses KF. Magnification, $\times 600$.

saged at a high cell density $\left(>5 \times 10^{6}\right.$ cells $/ 75 \mathrm{~cm}^{2}$ flask) that ensured cultures that consisted predominantly of EC-like cells. To induce their differentiation, $10^{6}$ NT2/D1 cells were seeded $/ 75 \mathrm{~cm}^{2}$ flask in medium containing $10^{-5} \mathrm{M}$ all-trans retinoic acid (Eastman Kodak) diluted from a $10^{-2} \mathrm{M}$ stock solution in dimethylsulfoxide, as previously described
(Andrews, 1984). These cultures were fed weekly with fresh medium containing retinoic acid until, after 3 weeks, the cells were harvested with $0.25 \%$ trypsin: $2 \mathrm{~mm}$ EDTA, reseeded at $5 \times 10^{6} \mathrm{cells} / 75 \mathrm{~cm}^{2}$ flask in the absence of retinoic acid, and maintained for a further 3-4 weeks. Whereas neuronal cells were evident $14-17 \mathrm{~d}$ following exposure to 
retinoic acid, the latter technique generally allowed the development of cultures containing a high proportion of neuronal cells, which formed extensive networks throughout the culture.

\section{Preparation of enriched human NF from spinal cord and cytoskeletal fractions from $N T 2 / D l$ cells}

Human NF proteins were prepared from the spinal cord of a 4-hr postmortem infant by a simple one-step procedure, as reported previously (Schlaepfer et al., 1984). In brief, the spinal cord was desheathed and homogenized in a 10-fold dilution (wt/vol) of homogenizing buffer $(1 \%$ Triton X-100, 1 м sucrose, 2.5 mм EDTA, 2.5 mм EGTA, $100 \mathrm{~mm}$ $\mathrm{NaCl}$, and $20 \mathrm{~mm}$ sodium phosphate buffer, $\mathrm{pH} 7.2$ ). Homogenates were centrifuged in a Beckman Ti45 rotor at 35,000 rpm for $6 \mathrm{hr}$. The floating lipid pad was removed and the soluble proteins in the supernatant discarded. The Triton-insoluble pellet was homogenized once again with the homogenizing buffer and centrifuged as described above for 12-16 $\mathrm{hr}$, to eliminate any residual myelin and lipid. Finally, the pellet was washed free of Triton and sucrose, using the homogenizing buffer described above, minus Triton and sucrose. After a brief centrifugation, the pellet was solubilized in Laemmli sample buffer for electrophoresis (Laemmli, 1970; see below).

Retinoic acid-treated and untreated NT2/D1 cells were washed three times with PBS containing $2.5 \mathrm{~mm}$ EGTA. Five $\mathrm{ml}$ of another homogenizing buffer ( $1 \%$ Triton X-100, $0.6 \mathrm{M} \mathrm{KCl}, 5 \mathrm{~mm}$ EGTA, 2 mM phenylmethyl sulfonyl fluoride (PMSF), $1 \mathrm{mg} / \mathrm{ml} p$-tosyl-L-arginine methyl ester (TAME), $10 \mathrm{mM} \mathrm{MgCl}$ and $0.5 \mathrm{mg} / \mathrm{ml} \mathrm{DNase}$ in PBS) was added to the cultures. The cells were then scraped from the tissue culture flasks and homogenized with ground-glass Duall homogenizers, after which the homogenates were allowed to stand for $30 \mathrm{~min}$ at $4^{\circ} \mathrm{C}$ to eliminate DNA. The homogenates were then centrifuged at $100,000 \times g$ for 60 $\mathrm{min}$. The soluble proteins were decanted and the Triton-insoluble pellets were washed in homogenizing buffer lacking Triton, $\mathrm{MgCl}_{2}$ and DNase. These insoluble cytoskeletal fractions were solubilized in freshly prepared Laemmli sample buffer, boiled for $10 \mathrm{~min}$, centrifuged in a microfuge, and immediately subjected to PAGE. The Triton-soluble proteins were recovered by precipitation with a 10 -fold dilution (vol/vol) of ice-cold acetone. These precipitates were washed twice with fresh, cold acetone and dissolved in Laemmli sample buffer.

\section{Antibodies to IF proteins}

The antibodics used, and thcir spccificitics, arc shown in Tablc 1. All of the MAs to NF were prepared using gel-excised NF proteins purified from bovine, human, or rat spinal cord as the immunogens. Rat polyclonal antisera were obtained from immunized rats prior to the removal of their spleens for fusion. MAs were prepared by fusing rat spleen cells (for bovine and human NF immunogens) or mouse spleen cells (for rat NF immunogens) with mouse myeloma cells (SP2/AG-14), as previously described (Lee et al., 1982b, 1984). MAs to GF and VF proteins have also been prepared following similar procedures, and their specificities have been described (Lee et al., 1984). Chrome 1, an anti-KF MA (Benham et al., 1983) specific for the 45,000 Da subunit of KF (R. Clark and I. Damjanov, personal communication), anti-cytokeratin (PKKI), and anti-vimentin MAs, purchased from Lab Systems (Chicago, IL), were also used.

\section{Immunofluorescence}

Differentiated and EC cultures were grown on glass coverslips, fixed with acetone, and stained for indirect immunofluorescence, as described previously (Andrews, 1984). Fluorescein- or rhodamine-labeled antimouse Ig or anti-rat Ig antibodies were purchased from Cappel Laboratories (Cochranville, PA). Double indirect immunofluorescence studies were conducted using fluorescein-labeled anti-rat Ig antibodies, absorbed with mouse $\mathrm{Ig}$, and rhodamine-labeled anti-mouse Ig, absorbed with rat Ig, to eliminate cross-reactivity between these two species.

\section{Gel electrophoresis and immunoblots}

All samples were electrophoresed on $0.75 \mathrm{~mm}$ slab gels of $7.5 \%$ acrylamide using the Laemmli gel system (Laemmli, 1970). Transfer of proteins from acrylamide gels to nitrocellulose paper was conducted according to the methods of Towbin et al. (1979), as modified by Lee et al. (1984). Protcin transfer was carricd out clectrophoretically at $0.3 \mathrm{~A}$ and $12 \mathrm{~V}$ for $45 \mathrm{~min}$ with a horizontal apparatus in Tris-glycine buffer containing $20 \%$ methanol.

The immunoblot procedure to detect bands recognized by the specific binding of polyclonal antisera and MAs to various IF proteins was undertaken (see Lee et al., 1984). Strips of nitrocellulose paper replicas of the gels were blocked for 2 to $4 \mathrm{hr}$ with Tris buffer and 1\% BSA, which was followed by overnight incubation in the appropriate antibodies. The strips were then washed, and bound antibody was labeled with peroxidase-conjugated rabbit anti-rat IgG (Cappel Laboratories) at a dilution of $1: 800$. Peroxidase in the second antibody was localized using an incubation medium containing 4-chloro-1-naphthol and hydrogen peroxide using the method of Hawkes et al. (1982), as modified by Lee et al. (1984).

\section{Results}

\section{Indirect immunofluorescence studies}

When cultures of undifferentiated EC stem cells were studied, most of the cells showed reactivity with the anti-KF MAs, and a few with the anti-VF MAs. No reactivity was detected with either anti-GF or any of the anti-NF subunit-specific MAs. Using polyclonal antisera and MAs that recognize epitopes on the three different NF subunits (Table 1), we detected each NF subunit in a few cells with a non-neuronal morphology as early as $2 \mathrm{~d}$ after retinoic acid treatment of the undifferentiated EC cells. Both NF1 70 and NF70 stained intensely, whereas NF195 stained only weakly at this time. At successive points in time, the proportion of NF-positive cells increased, and the reactivity with anti-NF195 MA became stronger. No evidence of cells expressing NF170 but not NF195 was found: in double-labeled immunofluorescence (Fig. 1, $A, B$ ), the immunostaining of RMDO20 (an MA raised in mouse, recognizing human NF195) colocalized with HO14 (an MA raised in rat to human NF170).

By 2 weeks in retinoic acid, almost all of the NF-positive cells $(>95 \%$ ) were found to be devoid of $\mathrm{KF}$ (Fig. 1, C,D), although $\mathrm{KF}$ was detected in many of the cells that were NF-negative (Fig. $1 D$ ), as determined by two-color indirect immunofluorescence. However, during the initial period of retinoic acid treatment (2-7 d), almost all (approximately 95\%) the NF-positive cells also contained KF although, again, most of the cells that reacted with anti-KF MA did not react with anti-NF MA (Fig. $1, E, F)$. At no time was vimentin detected in NF-positive cells (data not shown).

During this study it was noted that the size of cell bodies stained with anti-NF MAs varied (cf. Fig. 1, $A, C$, and $E$ ) but that this size variation was independent of the length of retinoic acid treatment. However, the length of axon-like processes stained with anti-NF MAs appeared to be directly related to time in retinoic acid. Cells with a neuronal morphology were first observed in cultures of NT2/D1 cells 14-17 d following first exposure to retinoic acid, and developed extensive axonlike processes during subsequent culture in the absence of retinoic acid. MAs to all three NF subunits, as well as polyclonal antisera to NF, reacted with these neuron-like cells in the same manner, and both the perikarya and the processes of these cells stained intensely (Fig. 2). MAs to the largest NF subunit (NF195) were previously shown to stain similar neuron-like cells using the indirect immunofluorescence technique (Andrews, 1984).

Many cells with a non-neuronal morphology and lacking NF were also present in NT2/D1 cultures exposed to retinoic acid, and we have previously shown that almost none of these are persisting EC cells (Andrews, 1984). More than 50\% of these differentiated cells were positive for VF, but the neuron-like cells were completely negative for VF (Fig. 3, $A, B$ ). Also, a substantial number of the non-neuron-like cells continued to show immunoreactivity with the anti-KF MAs (Fig. 3, $C, D$ ). Anti-GF MAs did not react with any of the cells.

\section{Electrophoretic and immunoblot analyses}

To confirm the specificity of the reactions detected by indirect immunofluorescence studies, IFs were extracted and analyzed by SDS-PAGE and immunoblotting. Figure 4 shows the protein 

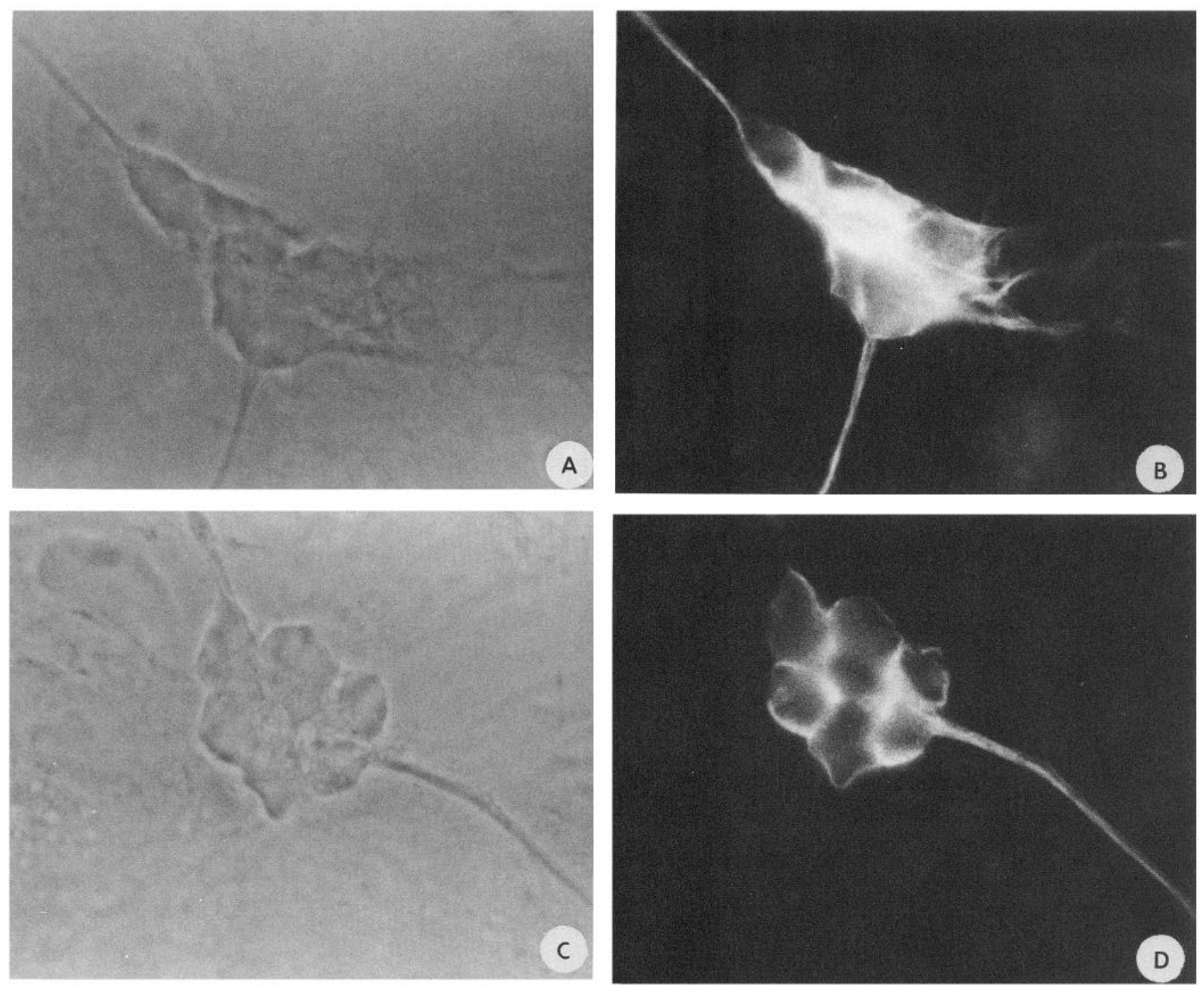

Figure 2. Indirect immunofluorescence staining of NT2/D1-derived neuron-like cells with anti-NF MAs. These cultures were obtained by preexposing NT2/D1 EC cells to $10^{-5} \mathrm{M}$ retinoic acid for 3 weeks, followed by growth on glass coverslips for 4 weeks in the absence of retinoic acid. $A$, Phase contrast of a cluster of neuron-like cells. B, Fluorescence staining of NF70 in the same cells with antibody Sd112. $C$, Phase contrast of another cluster of neuron-like cells. $D$, Fluorescence staining of both NF70 and NF170 with an MA (Oc25) that recognizes a shared epitope. Note that non-neuronal cells did not stain. Magnification, $\times 600$.

profiles of the cytoskeletal fractions isolated from the NT2/D1 EC cells (lane 1) and their retinoic acid-induced, differentiated derivatives (lane 2). For comparison, enriched human NF fractions were similarly separated (lane 3 ). It is evident that NF proteins were not the major components in the cytoskeletal fractions obtained from the differentiated cultures. Major bands from both sets of cultures comigrated with VF, but it is unclear from the Coomassie blue-stained gel whether these intense bands were indeed VF proteins.

Figure 5 illustrates the results of immunoblot studies on these preparations. In each of the subsets $(A-G)$, lane 1 shows the patterns of the different IF proteins detected in separated, enriched human NF fractions by the various MAs. Lanes 2 and 3 show the immunoreactivities using the same MAs on separated cytoskeletal fractions isolated from differentiated and EC cells, respectively. All three NF subunits were detected in the differentiated cells but not in the EC cells (Fig. 5, $A-E$ ). NF195 sometimes appeared as a doublet in both human NF and the differentiated cell preparations (Fig. $5 A$ ). This observation was not consistently seen, however. Figure $5 B$ shows the presence of NF195 and nontriplet NF proteins with molecular weights between 57,000 and 65,000 . We have also observed such nontriplet NF proteins derived from NF195 in transected rat sciatic nerves (Schlaepfer et al., 1984). Interestingly, these nontriplet NF proteins were the only NF immunoreactive polypeptides detected in the Triton-soluble fractions prepared from differentiated EC cells; no other IF proteins were detected from this fraction (data not shown). Figure $5 C$ demonstrates the presence of NF170 using a MA that recognizes NF170 exclusively. Figure $5 D$, on the other hand, illustrates the immunobands of NF170 and NF70 in lanes 1 and 2 as recognized by a MA that detects a determinant shared by NF170 and NF70. Figure $5 E$ shows the NF70 immunoband in the differentiated cells, as detected by an anti-NF70-specific MA. Finally, Figure $5 F$ shows the presence of VF in all three preparations (lanes 1-3). It is obvious that VF proteins have undergone proteolysis, which is commonly seen (Ciesielski-Treska et al., 1984). As expected, KF was detected in both retinoic acid-treated and untreated EC cells but not in the human NF preparations (Fig. $5 G$ ). GF protein was not detected under any culture condition (data not shown). 

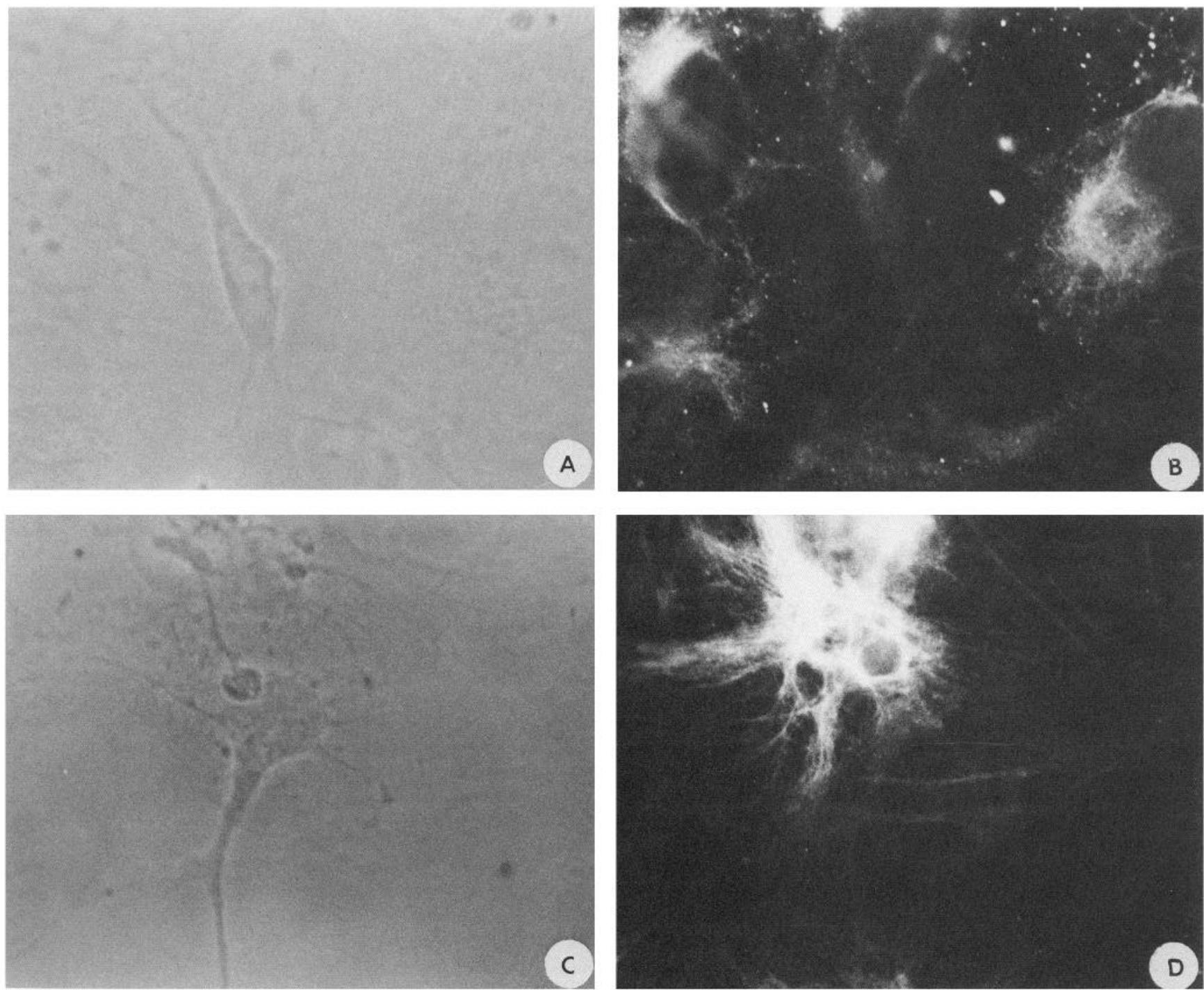

Figure 3. Indirect immunofluorescence staining of differentiated NT2/D1 cells with anti-KF $(A$ and $B)$ and anti-VF MAs. Cells were grown as in Fig. 2. $A$ and $C$, Phase contrast of a neuron-like cell in the presence of non-neuronal cells. $B, \mathrm{KF}$ staining of non-neuronal cells but not the neuronlike cell. $D$, VF staining of non-neuronal cells but not the neuron-like cell. Magnification, $\times 600$.

\section{Discussion}

Many cell lines have been isolated from human teratocarcinomas and may contain EC stem cells (Andrews, 1983), but few appear to have retained a capacity for even limited differentiation (e.g., Andrews et al., 1982; McIlhinney et al., 1983). So far, only human EC cells isolated from the TERA-2 cell line, such as clone NT2/D1, have been shown to differentiate into a variety of somatic cell types (Andrews et al., 1984; Thompson et al., 1984). As in other studies of human EC cells (Damjanov and Andrews, 1983; Damjanov et al., 1984), we have found that $\mathrm{KF}$ is the predominant IF of these stem cells, whereas VF is present in only a subpopulation of cells in uninduced cultures. These VF-positive cells probably represent the few spontaneously differentiated cells that are inevitably present in EC cultures (see also Damjanov and Andrews, 1983). This contrasts with the situation in mouse EC cells, which express VF but not $\mathrm{KF}$, and only express KF when induced to differentiate, for example, by treatment with retinoic acid (Benham et al., 1983; Paulin et al., 1982; Ramaekers et al., 1984). Human and mouse EC cells also differ in a number of other aspects, and we have speculated that they may represent different populations of embryonic stem cells (Andrews et al., 1983).
We have previously shown that NT2/D1 EC cells are induced by retinoic acid to differentiate into various cell types that include neurons (Andrews, 1984). Our present results demonstrate that the EC stem cells do not express any of the NF-subunit proteins, whereas the neurons derived following induction with retinoic acid express all three NF subunits, but not VF and KF. Thus, the appearance of NF proteins in the neuronal derivatives must involve induction of their synthesis. Further, since, during the early stage of retinoic acid-induced differentiation, almost all cells expressing NF also expressed KF, but not VF, we conclude that the immediate progenitor cells of these neurons are KF-positive. Other differentiated cells derived from NT2/D1 EC cells do express VF and KF, but their identity remains unknown. None, however, expresses glial filaments. Similarly, EC cells in the independent series of TERA-2-derived clones studied by Thompson et al. (1984) were also found to differentiate into neurons but not glial cells. Additional efforts to induce GF-positive cells, for example, by glial maturation factor (Lim, 1980), have so far proved unsuccessful (P. W. Andrews and R. Lim, unpublished observations). Since glial cells and neurons have a common embryological origin in the neural ectoderm, it is unclear why TERA-2 EC stem cells apparently 
Table 1. Monoclonal antibodies to intermediate filaments used in this study

\begin{tabular}{lll} 
Antibody & $\begin{array}{l}\text { Specificity } \\
\text { in human } \\
\text { cells }^{a}\end{array}$ & Reference \\
\hline Oc19 & NF195 & Lee et al. (1982b) \\
Oc92 & NF195 & Lee et al. (1982b) \\
Ta51 & NF195 & Lee et al. (1982b) \\
Ta52 & NF195 $^{b}$ & Schlaepfer et al. (1984) \\
RMDO20 & NF195 $^{c}$ & Lee et al. (unpublished results) \\
Oc109 & NF195; NF170 & Lee et al. (1982b) \\
Ta22 & NF195; NF170 & Lee et al. (1982b) \\
Ta54 & NF170 & Lee et al. (1982b) \\
HO14 & NF170 & Lee et al. (unpublished results) \\
HO45 & NF170 & Lee et al. (unpublished results) \\
Oc25 & NF170; NF70 & Lee et al. (1982b) \\
Sd112 & NF70 & Lee et al. (1982b) \\
Ta66 & NF70 & Lee et al. (1982b) \\
RMS14 & NF70 & Lee et al. (unpublished results) \\
PKK1 & KF44, KF46, & Lab Systems, Inc., \\
& KF52, KF54 & Chicago, IL \\
Chrome 1 & KF45 & Benham et al. (1983); \\
& & Damjanov and Clark \\
(personal communication) & Lab Systems, Inc., Chicago, IL \\
Anti-vimentin & VF & Lee et al. (1984) \\
$1 \cdot 3 H 9$ & VF & Lee et al. (1984) \\
2.2B10 & GF & \\
\hline
\end{tabular}

a The specificity for human IF is not necessarily identical to the specificity for IF from other species (Lee, unpublished data).

${ }^{b}$ Ta52 also recognized NF nontriplet proteins in immunoblot analysis.

- RMDO20 and RMS14 were raised using rat NF as immunogen.

${ }^{d}$ Ta54 and Ta66 react with SDS-denatured NF subunit proteins in immunoblot analysis but not in indirect immunofluorescence of fixed cells in situ.

' Both $\mathrm{HO} 14$ and $\mathrm{HO} 45$ were raised using human NF170 as immunogen.

only give rise to the neuronal lineage. The absence of GF protein in any of the NT2/D1 EC cell derivatives contrasts with observations of clinical specimens of human teratomas in situ, which often contain cells that express such differentiated neural antigens as NF, GF, and myelin basic protein (Trojanowski and Hickey, 1984). Whether such human teratomas generally express all three NF subunits in situ remains to be determined.

The early stages of neuronal differentiation during embryogenesis are poorly understood. In part, this reflects the lack of probes to follow the fate of differentiating cells and the inaccessibility of embryonic cells in situ to experimental manipulation, especially in the case of human development. However, a number of studies have shown that expression of the different classes of IF proteins, including NF proteins, is developmentally regulated. During embryogenesis, the earliest IF protein detectable in preimplantation mouse embryos is a KF polypeptide (Jackson et al., 1980). Studies of the chick neural tube at later stages of development have shown that VF protein is expressed by dividing cells that, when they become committed neurons, express NF proteins and cease expressing VF protein (Tapscott et al., 1981). Nevertheless, in some mature neurons of the mouse, VF continues to be seen (Drager, 1983; Shaw and Weber, 1984). Fewer data are available concerning the expression of different classes of IF proteins in the developing human nervous system. Our present results with differentiating human teratocarcinoma cells indicate that some human neurons may arise from KFrather than VF-positive precursors. It is also noteworthy that the progenitors of human lung neuroendocrine cells have been

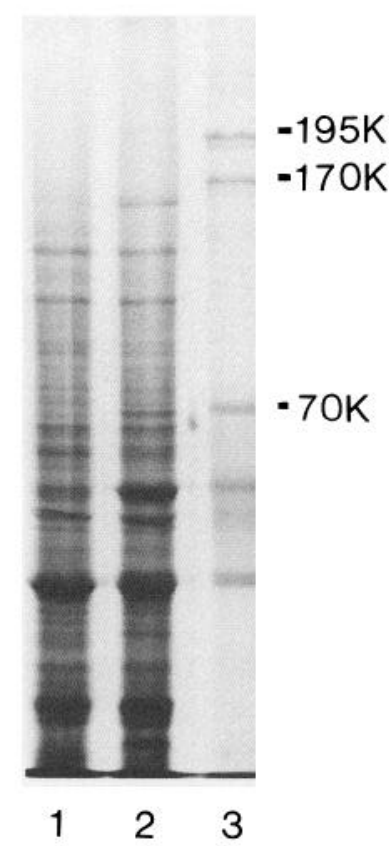

Figure 4. Electrophoretic profile of proteins in Triton-insoluble pellets from NT2/D1 stem cells (lane 1); from the retinoic acid-treated NT2/ D1 differentiated cells (lane 2); and from human spinal cord containing NF triplet proteins of $M_{r} 195,000,170,000$, and 70,000 (lane 3). All proteins were electrophoresed on the same $7.5 \%$ acrylamide gel and stained with Coomassie blue.

shown to express both NF and KF, but to express either NF or KF only after terminal differentiation (Bergh et al., 1984).

An in vitro model system for developing neurons presents clear advantages over in situ studies for addressing certain issues, such as the identification of factors that promote or regulate differentiation. Recently, we have shown that NGF can induce the expression of NF proteins in clonal rat pheochromocytoma cell line PC12 (Lee and Page, 1984; Lee et al., 1984), which also undergoes a form of differentiation. Like EC cells, PC1 2 cells are neoplastic cells. However, the NGF-induced derivatives of the PC12 cells are different from the neurons that arise from the human EC cells studied here in that they contain abundant amounts of VF, in addition to the two smaller NF subunits, and lack, or contain only very minute quantities of, the largest NF subunit (Lee, 1985). Another striking difference between the IF structures of PC12 cells and the EC cell-derived neurons is the arrangement of IF structures in these cells; IF exist in "balllike" aggregates in PC12 cells, but are finely filamentous in the EC cell-derived neurons. We have speculated that this arrangement may reflect abnormalities in NF-subunit synthesis, assembly, or turnover in PC12 cells (Lee and Page, 1984; Lee, 1985). It is possible that the lack of normal NF195 in PC12 cells, and its presence in differentiated EC cells, account for the presence of "ball-like" IF aggregates in the former and their absence in the latter cells. This would be consistent with the view that normal NF195 performs a cross-linking function that may determine the relationship of the NF subunits to each other and to other cytoplasmic organelles (Hirokawa et al., 1984). Comparisons of the NF proteins in these two cell lines may thus provide insights into the mechanism leading to the formation of the abnormal "ball-like" arrays of IF seen in PC12 cells and other tumors (Trojanowski et al., 1984b), as well as into the function(s) subserved by normal NF structures.

The appearance of a normal spectrum of NF proteins during differentiation of NT2/D1 EC cells into neurons suggests that this process resembles that of neuronal maturation during em- 
A

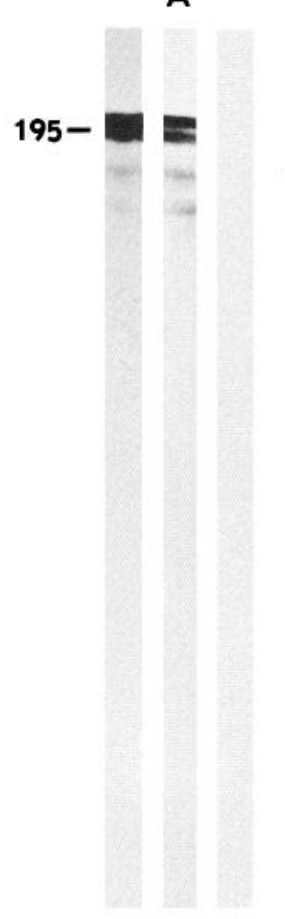

B

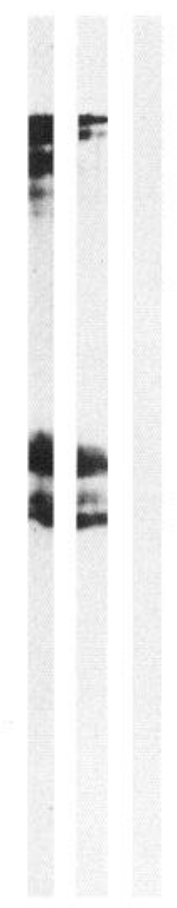

C

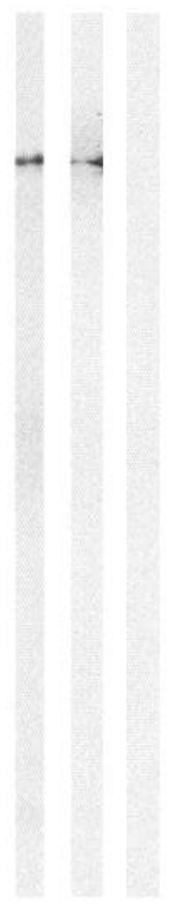

D

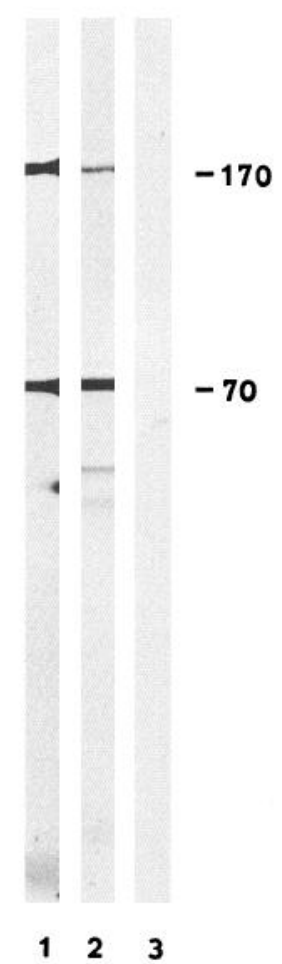

E

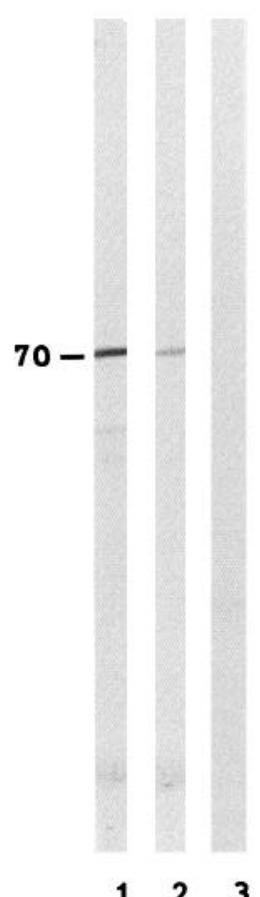

$\mathbf{F}$

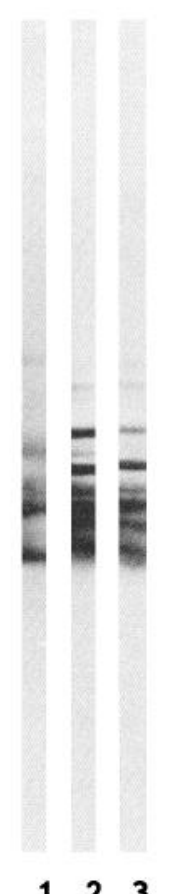

G

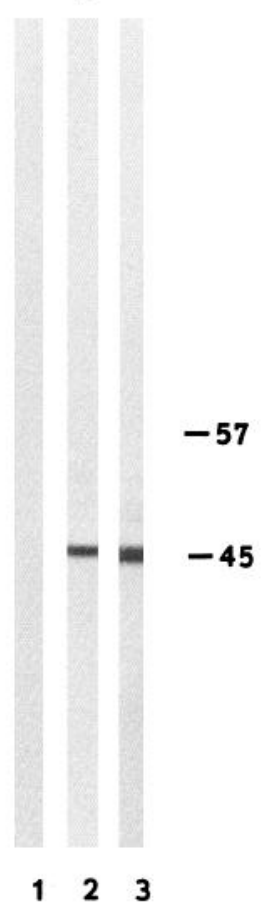

Figure 5. Immunoblots of Tritoninsoluble proteins from human spinal cord (lanes 1), retinoic acid-treated differentiated NT2/D1 cells (lanes 2) and NT2/D1 stem cells (lanes 3 ). Different MAs were used: $A$, Ta51, specific for NF195 in human; $B$, Ta52, specific for NF195 and NF nontriplet proteins; $C$, Ta54, specific for NF170; $D$, Oc25, detecting on epitope shared by NF70 and NF170; $E$, Ta66, specific for NF70; $F, 1.3 \mathrm{H} 9$, specific for VF; $G$, Chrome 1 , specific for the $M_{r}$ 45,000 polypeptide of KF. NF triplet proteins: $M, 195,000,170,000$, and 70,000; VF protein: $M_{r} 57,000$; and KF protein: $M, 45,000$. bryogenesis, and that NT2/D1 EC cells will provide a useful new model for investigating human neural development. Comparative studies of the IF proteins in EC cells grown under different conditions that induce differentiation, and the IF proteins present in human teratomas in situ, may contribute to a better understanding of the events and growth factor(s) that determine differentiation in human teratomas. Such studies should also provide information about the factors that control normal differentiation and the regulation of IF protein expression.

\section{References}

Andrews, P. W. (1983) The characteristics of cell lines derived from human germ cell tumors. In The Human Teratomas: Experimental 
and Clinical Biology, I. Damjanov, B. B. Knowles, and D. Solter, eds., pp. 285-311, Humana, Clifton, NJ.

Andrews, P. W. (1984) Retinoic acid induces neuronal differentiation of a cloned human embryonal carcinoma cell line in vitro. Dev. Biol. 103: 298-293.

Andrews, P. W., I. Damjanov, D. Simon, G. S. Banting, C. Carlin, N. Dracopoli, and J. Fogh (1984) Pluripotent embryonal carcinoma clones derived from the human teratocarcinoma cell line Tera-2. Lab. Invest. 50:147-162.

Andrews, P. W., P. N. Goodfellow, L. H. Shevinsky, D. L. Bronson, and B. B. Knowles (1982) Cell surface antigens of a clonal human embryonal carcinoma cell line: Morphological and antigenic differentiation in culture. Int. J. Cancer 29: 523-531.

Andrews, P. W., P. N. Goodfellow, and I. Damjanov (1983) Human teratocarcinoma cells in culture. Cancer Surv. 2: 41-73.

Benham, F. J., M. V. Wiles, G. Banting, P. W. Andrews, and P. N. Goodfellow (1983) Human-mouse teratocarcinoma hybrids. A tool for analysis of gene activity in early human development. In The Human Teratomas: Experimental and Clinical Biology, I. Damjanov, B. B. Knowles, and D. Solter, eds., pp. 313-341, Humana, Clifton, NJ.

Bennett, G. S., S. J. Tapscott, C. Dilullo, and H. Holtzer (1984) Differential binding of antibodies against the neurofilament triplet proteins in different avian neurons. Brain Res. 304: 291-302.

Bergh, J., K. Nilsson, D. Dahl, L. Andersson, I. Virtanen, and V.-P. Lehto (1984) Expression of intermediate filaments in established human lung cancer cell lines. An indicator of differentiation and derivation. Lab. Invest. 51: 307-316.

Ciesielski-Treska, J., J.-F. Goetschy, and D. Aunis (1984) Proteolytic degradation of vimentin and glial acid protein in rat astrocytes in primary culture. Eur. J. Biochem. 138: 465-471.

Dahl, D. (1983) Immunohistochemical differences between neurofilaments in perikarya, dendrites and axons. Immunofluorescence study with antisera raised to neurofilaments in perikarya, dendrites and axons. Exp. Cell Res. 149: 397-408.

Damjanov, I., and P. W. Andrews (1983) Ultrastructural differentiation of a clone human embryonal carcinoma cell line in vitro. Cancer Res. 43: 2190-2198.

Damjanov, I., R. K. Clark, and P. W. Andrews (1984) Cytoskeleton of human embryonal cells. Cell Differ. 15: 133-139.

Drager, U. C. (1983) Coexistence of neurofilaments and vimentin in a neuron of adult mouse retina. Nature 303: 169-171.

Ilawkes, R., E. Nidaz, and B. Gordon (1982) A dot-immunoblot assay for monoclonal and other antibodies. Anal. Biochem. 119: 142-147.

Hirokawa, N., M. A. Glicksman, and M. B. Willard (1984) Organization of mammalian neurofilament polypeptides within the neuronal cytoskeleton. J. Cell Biol. 98: 1523-1536.

Jackson, B. W., C. Grund, E. Schmid, K. Burki, W. W. Franke, and K. Illmensee (1980) Formation of cytoskeletal elements during mouse embryogenesis. I. Intermediate filaments of the cytokeratin type and desmosomes in preimplantation embryos. Differentiation 17:161179

Jones-Villeneuve, E. M. V., M. W. McBurney, K. A. Rogers, and V. I. Kalnins (1982) Retinoic acid induces embryonal carcinoma cells to differentiate into neurons and glial cells. J. Cell Biol. 94: 253-262.

Laemmli, U. K. (1970) Cleavage of structural proteins during the assembly of bacteriophage T4. Nature 227: 680-685.

Lazarides, E. (1982) Intermediate filaments: A chemically heterogeneous, developmentally regulated class of proteins. Annu. Rev. Biochem. 51: 219-250.

Lee, V. M.-Y. (1985) Neurofilament protein abnormalities in $\mathrm{PC1} 2$ cells: Comparison with neurofilament proteins of normal cultured rat sympathetic neurons. J. Neurosci. 5: 3039-3046.
Lee, V. M.-Y., and C. Page (1984) The dynamics of nerve growth factor-induced neurofilament and vimentin filament expression and organization in PC12 cells. J. Neurosci. 4: 1705-1714.

Lee, V. M.-Y., C. D. Page, H.-L. Wu, and W. W. Schlaepfer (1984) Monoclonal antibodies to gel excised glial filament protein and their reactivity with other intermediate filament proteins. J. Neurochem. 42: 25-32.

Lee, V., J. Q. Trojanowski, and W. W. Schlaepfer (1982a) Induction of neurofilament triplet proteins in PC12 cells by nerve growth factor. Brain Res. 238: 169-180.

Lee, V. M.-Y., H.-L. Wu, and W. W. Schlaepfer (1982b) Monoclonal antibodies recognize individual neurofilament triplet proteins. Proc. Natl. Acad. Sci. USA 79: 6089-6092.

Lim, R. (1980) Glial maturation factor. Curr. Top. Dev. Biol. 16: 305322.

Martin, G. R. (1980) Teratocarcinomas and mammalian embryogenesis. Science 209: 768-776.

McIlhinney, R. A. J., S. Patel, and P. Monaghan (1983) Effects of $12-$ O-tetradecanylphorbol 13-acetate(TPA) on a clonal human teratomaderived embryonal carcinoma cell line. Exp. Cell Res. 144: 297-311.

Paulin, D., H. Jakob, F. Jacob, K. Weber, and M. Osborn (1982) In vitro differentiation of mouse teratocarcinoma cells monitored by intermediate filament expression. Differentiation 22: 90-99.

Ramaekers, F., H. Schaap, M. Mulder, A. Huysmans, and P. Vooijs (1984) Cytokeratin filament expression during in vitro teratocarcinoma ccll differentiation as described by monoclonal antibody. Cell Biol. Int. Rep. 8: 721-730.

Schlaepfer, W. W., C. Lee, J. Q. Trojanowski, and V. M.-Y. Lee (1984) Persistence of immunoreactive neurofilament protein breakdown products in transected rat sciatic nerve. J. Neurochem. 43: 857-864.

Shaw, G., and K. Weber (1983) The structure and development of the rat retina: An immunofluorescence microscopical study using antibodies specific for intermediate filament proteins. Eur. J. Cell Biol. 30: 219-232.

Solter, D., and I. Damjanov (1979) Teratocarcinoma and the expression of oncodevelopmental genes. Meth. Cancer Res. 18: 277-332.

Stevens, L. C. (1967) The biology of teratomas. Adv. Morphol. 6: 131.

Tapscott, S. J., G. S. Bennett, and H. Holtzer (1981) Neuronal precursor cclls in chick neural tube express neurofilament proteins. Nature 292: 836-838.

Thompson, S., P. L. Stern, M. Webb, F. S. Walsh, W. Engstrom, E. P. Evans, W.-K. Shi, B. Hopkins, and C. F. Graham (1984) Cloned human teratoma cells differentiate into neuron-like cells and other cell types in retinoic acid. J. Cell Sci. 72: 37-64.

Towbin, H., T. Staehelin, and J. Gordon (1979) Electrophoretic transfer of proteins from acrylamide gels to nitrocellulose sheets: Procedure and some applications. Proc. Natl. Acad. Sci. USA 76: 4350-4354.

Toyoshima, I., M. Satake, and T. Miyatake (1984) Differences in the neurofilament proteins between the perikarya and axon of the bovine spinal ganglion. Biomedical Res. 5: 459-464.

Trojanowski, J. Q., and W. F. Hickey (1984) Human teratomas express differentiated neural antigens: An immunohistochemical study with antincurofilament, anti-glial filament and anti-myeline basic protein monoclonal antibodies. Am. J. Pathol. 115: 383-389.

Trojanowski, J. Q., D. Gordon, M. Obrocka, and V. M.-Y. Lee (1984a) The developmental expression of neurofilament and glial filament proteins in the human pituitary gland: An immunohistochemical study with monoclonal antibodies. Dev. Brain Res. 13: 229-239.

Trojanowski, J. Q., V. M.-Y. Lee, and W. W. Schlaepfer (1984b) An immunohistochemical study of human central and peripheral nervous system tumors using monoclonal antibodies against neurofilaments and glial filaments. Human Pathol. 15: 248-257. 